\title{
RANDOM FIXED POINT THEOREMS FOR CONTRACTIVE TYPE MULTIFUNCTIONS
}

\author{
GHULAM MUSTAFA
}

(Received 3 April 2003; revised 13 August 2003)

Communicated by A. J. Pryde

\begin{abstract}
Some new random coincidence point and random fixed point theorems for multivalued mappings in separable complete metric spaces are proved. The results presented in this paper are the stochastic versions of corresponding results of Chang and Peng and extend the result of the author.
\end{abstract}

2000 Mathematics subject classification: primary 5H25, 7H10.

Keywords and phrases: multifunction, random fixed point, random coincidence point.

\section{Introduction and preliminaries}

In order to generalise the well-known contraction principle of Banach to multivalued functions and random fixed point theorems, many authors ([1,5,7-11]) introduced more general contractive inequalities. We intend to consider a class of generalised contractions that includes the classes considered in ([1,5,7-11]) and that enables us to prove a more general random fixed point theorem for multifunctions.

Throughout this paper $(X, d)$ is a separable complete metric space, $\mathbb{R}^{+}=[0, \infty)$ and $(\Omega, \delta)$ is a measurable space. Let $2^{X}$ be the family of all subsets of $X, C B(X)$ denote the family of all nonempty closed bounded subsets of $X$ and $C C(X)$ denote the family of all nonempty compact subsets of $X$. For any nonempty subsets $A, B$ of $X$, we denote

$$
\begin{gathered}
d(x, A)=\inf \{d(x, a): a \in A\} \quad(x \in X), \\
d(A, B)=\inf \{d(a, b): a \in A, b \in B\}, \\
H(A, B)=\max \left\{\sup _{a \in A} d(a, B), \sup _{b \in B} d(b, A)\right\}
\end{gathered}
$$

(C) 2005 Australian Mathematical Society 1446-7887/05 $\$ A 2.00+0.00$ 
and $H(\cdot, \cdot)$ is called the Hausdorff metric on $C B(X)$.

A mapping $\mu: \Omega \rightarrow 2^{X}$ is called measurable if for any open subset $C$ of $X$, $\mu^{-1}(C)=\{w \in \Omega: \mu(w) \cap C \neq \emptyset\} \in \delta$. A mapping $\xi: \Omega \rightarrow X$ is said to be measurable selector of a measurable mapping $\mu: \Omega \rightarrow 2^{X}$ if $\mu$ is measurable and for any $w \in \Omega, \xi(w) \in \mu(w)$. A mapping $f: \Omega \times X \rightarrow X$ is called a random operator if for any $x \in X, f(\cdot, x)$ is measurable. A mapping $T: \Omega \times X \rightarrow C B(X)$ is called a multifunction if for every $x \in X, T(\cdot, x)$ is measurable. A measurable mapping $\xi: \Omega \rightarrow X$ is called a random fixed point of a multifunction (random operator) $T: \Omega \times X \rightarrow C B(X)(f: \Omega \times X \rightarrow X)$ if for every $w \in \Omega, \xi(w) \in T(w, \xi(w))$ $(f(w, \xi(w))=\xi(w))$. A measurable mapping $\xi: \Omega \rightarrow X$ is a random coincidence point of $T: \Omega \times X \rightarrow C B(X)$ and $f: \Omega \times X \rightarrow X$ if for every $w \in \Omega, f(w, \xi(w)) \in$ $T(w, \xi(w))$.

For the remaining part of this section $S, T: \Omega \times X \rightarrow C B(X)$ are multifunctions, $f: \Omega \times X \rightarrow X$ is a random operator and $\xi_{n}: \Omega \rightarrow C B(X)$ is a measurable mapping for each $n=0,1,2, \ldots$

For a map $\xi_{0}: \Omega \rightarrow X$, if there exists a sequence $\left\{\xi_{n}(w)\right\}$ such that $f\left(w, \xi_{n+1}(w)\right) \in$ $S\left(w, \xi_{n}(w)\right), f\left(w, \xi_{n+2}(w)\right) \in T\left(w, \xi_{n+1}(w)\right), n=0,1,2, \ldots$, then $O_{f}\left(\xi_{0}(w)\right)=$ $\left\{f\left(w, \xi_{n}(w)\right): n=1,2,3, \ldots\right.$ for each $\left.w \in \Omega\right\}$ is the orbit for $(S, T, f)$ at $\xi_{0}(w)$. If there exists a measurable map $\xi: \Omega \rightarrow X$ such that $f\left(w, \xi_{n}(w)\right) \rightarrow f(w, \xi(w))$ for all $w \in \Omega$, then $O_{f}\left(\xi_{0}(w)\right)$ converges in $X$. If $O_{f}\left(\xi_{n}(w)\right)$ converges in $X$, then $X$ is called $\left(S, T, f, \xi_{0}(w)\right)$-orbitally complete.

A function $\Psi\left(t_{1}, t_{2}, t_{3}, t_{4}, t_{5}\right): \mathbb{R}^{+5} \rightarrow \mathbb{R}^{+}$is said to satisfy the condition $(\Psi)$, if it is nondecreasing in each variable and there exists an increasing function $\Phi(t): \mathbb{R}^{+} \rightarrow$ $\mathbb{R}^{+}$satisfying the conditions (1) and (2) such that

$$
\Psi(t, t, t, a t, b t) \leq \Phi(t), \quad \text { for all } t \geq 0, \quad a+b=3, \quad a, b=1,2 .
$$

LEMMA 1.1 ([2]). Let $(X, d)$ be a metric space, $A \subset X$ a nonempty compact subset and $B \subset X$ a closed subset. If $d(A, B)=0$, then $A \cap B \neq \emptyset$.

LEMMA $1.2([13$, Theorem 1$])$. Let $\Phi: \mathbb{R}^{+} \rightarrow \mathbb{R}^{+}$be an increasing function such that

$$
\begin{aligned}
& \Phi(t+)<t \text { for all } t>0 \text { and } \\
& \sum \Phi^{n}(t) \text { is finite for all } t>0 .
\end{aligned}
$$

Then there exists a strictly increasing function $\phi: \mathbb{R}^{+} \rightarrow \mathbb{R}^{+}$such that

$$
\begin{gathered}
\Phi(t)<\phi(t) \quad \text { for all } t>0 \text { and } \\
\sum \phi^{n}(t) \quad \text { is finite for } t>0 .
\end{gathered}
$$


LEMMA 1.3 ([13]). (i) If $\Phi: \mathbb{R}^{+} \rightarrow \mathbb{R}^{+}$is strictly increasing and satisfies (2), then $\Phi$ satisfies (1).

(ii) Let $\Phi: \mathbb{R}^{+} \rightarrow \mathbb{R}^{+}$be increasing and satisfying (1). If $\sum \Phi^{n}\left(t_{1}\right)$ is convergent for some $t_{1}>0$, then (2) holds.

(iii) Let $\Phi: \mathbb{R}^{+} \rightarrow \mathbb{R}^{+}$be increasing and satisfying (1). If $t \leq \Phi(t)$ then $t=0$.

LEMMA 1.4 ([11]). If $S_{1}, S_{2}: \Omega \rightarrow C B(X)$ are measurable multifunctions, $s_{1}$ : $\Omega \rightarrow X$ is a measurable selector of $S_{1}$ and $\lambda: \Omega \rightarrow(1, \infty)$ is a measurable function then there exists a measurable selector $s_{2}: \Omega \rightarrow X$ of the multifunction $S_{2}$ such that $d\left(s_{1}(w), s_{2}(w)\right) \leq \lambda(w) H\left(S_{1}(w), S_{2}(w)\right)$.

LEMMA 1.5 ([11]). If $S: \Omega \rightarrow C B(X)$ is a continuous multifunction then $x \rightarrow$ $d(x, S(x))$ is a continuous real valued function.

\section{Main results}

Recently, Mustafa [8] gave the stochastic generalisation of the results of Kaneko and Sessa [4] and proved the following theorem:

THEOREM 2.1. Let $S, T: \Omega \times X \rightarrow C B(X)$ be two continuous multifunctions and let $f: \Omega \times X \rightarrow X$ be a random operator such that $S(w, X) \cup T(w, X) \subseteq$ $f(w, X)$ and for a measurable map $\xi_{0}: \Omega \rightarrow X, f(w, X)$ is $\left(S, T, f, \xi_{0}(w)\right)$-orbitally complete, for every $w \in \Omega$, and

$$
\begin{aligned}
H(S(w, x), T(w, y)) \leq & \alpha(w) \max \{d(f(w, x), f(w, y)), \\
& d(f(w, x), S(w, x)), d(f(w, y), T(w, y)), \\
& {[d(f(w, x), T(w, y))+d(f(w, y), S(w, x))] / 2\} }
\end{aligned}
$$

for all $x, y \in X$ and for all $w \in \Omega$, where $\alpha: \Omega \rightarrow(0,1)$ is a measurable map. Then there exists a random coincidence point of $S, T$ and $f$.

Let $S, T: \Omega \times X \rightarrow C B(X)$ and $F: \Omega \times X \rightarrow C C(X)$ be multifunctions such that

$$
\begin{aligned}
H(S(w, x), T(w, y)) \leq & \Phi(\max \{d(F(w, x), F(w, y)), \\
& d(F(w, x), S(w, x)), d(F(w, y), T(w, y)), \\
& {[d(F(w, x), T(w, y))+d(F(w, y), S(w, x))] / 2\}) }
\end{aligned}
$$

for all $x, y \in X$ and for all $w \in \Omega$, where $\Phi: \mathbb{R}^{+} \rightarrow \mathbb{R}^{+}$is an increasing function satisfying conditions (1) and (2).

As an improvement and generalisation of Theorem 2.1, we have the following 
THEOREM 2.2. Let $S, T: \Omega \times X \rightarrow C B(X)$ and $F: \Omega \times X \rightarrow C C(X)$ be multifunctions such that

(i) $S(w, \cdot), T(w, \cdot)$ are both continuous for all $w \in \Omega$;

(ii) $S(\cdot, x), T(\cdot, x)$ are both measurable for all $x \in X$;

(iii) $S(w, X) \cup T(w, X) \subset F(w, X), F(w, X)$ is closed;

(iv) $S, T$ and $F$ satisfy (5) for all $w \in \Omega$ and all $x, y \in X$.

Then there exists a measurable map $s: \Omega \rightarrow X$ such that

$$
F(w, s(w)) \cap S(w, s(w)) \cap T(w, s(w)) \neq \emptyset .
$$

PROOF. By Lemma 1.2, there exists a strictly increasing function $\phi: \mathbb{R}^{+} \rightarrow \mathbb{R}^{+}$ satisfying conditions (3) and (4). For any $x, y \in X$ and $w \in \Omega$, let us denote

$$
\begin{gathered}
A(x, y) \leq \max \{d(F(w, x), F(w, y)), d(F(w, x), S(w, x)), d(F(w, y), T(w, y)), \\
[d(F(w, x), T(w, y))+d(F(w, y), S(w, x))] / 2\} .
\end{gathered}
$$

Then (5) can be reduced to

$$
H(S(w, x), T(w, y)) \leq \Phi(A(x, y))
$$

Let $\varphi: \Omega \times X \rightarrow \mathbb{R}^{+}$be the function

$$
\varphi(w, x)=d(x, S(w, x)),(w, x) \in \Omega \times X .
$$

Since by (ii) $w \rightarrow S(w, x)$ is measurable for all $x \in X$ we conclude that $\varphi(\cdot, x)$ is measurable (see [3, Theorem 3.5]) and since $x \rightarrow S(w, x)$ is continuous for all $w \in \Omega$, we deduce from Lemma 1.5 that $\varphi(w, \cdot)$ is continuous for all $w \in \Omega$. Hence $\varphi: \Omega \times X \rightarrow \mathbb{R}^{+}$is a Caratheodory function. Therefore, if $u: \Omega \rightarrow X$ is a measurable mapping we also have that $w \rightarrow \varphi(w, u(w))$ is measurable (see [12]). If $\xi_{0}, \xi_{1}: \Omega \rightarrow X$ are measurable mappings and we consider the multifunction $S\left(\cdot, \xi_{0}(\cdot)\right): \Omega \rightarrow C B(X)$, then we deduce from the Kuratowski-Ryll Nardzewski Selection Theorem [6] that there is a measurable selector $s_{1}: \Omega \rightarrow X$ such that $s_{1}(w) \in S\left(w, \xi_{0}(w)\right)$ for all $w \in \Omega$. Applying Lemma 1.4 we find a measurable function $s_{2}: \Omega \rightarrow X$ such that $s_{2}(w) \in T\left(w, \xi_{1}(w)\right), w \in \Omega$.

For any measurable map $\xi_{0}: \Omega \rightarrow X$, since $S(w, X) \subset F(w, X)$, there exist measurable maps, say $\xi_{0}, \xi_{1}: \Omega \rightarrow X$ such that $F\left(w, \xi_{1}(w)\right) \cap S\left(w, \xi_{0}(w)\right) \neq \emptyset$. Let $s_{1}(w) \in F\left(w, \xi_{1}(w)\right) \cap S\left(w, \xi_{0}(w)\right)$, then we have

$$
\begin{aligned}
d\left(s_{1}(w), T\left(w, \xi_{1}(w)\right)\right) & \leq d\left(s_{1}(w), S\left(w, \xi_{0}(w)\right)\right)+H\left(S\left(w, \xi_{0}(w)\right), T\left(w, \xi_{1}(w)\right)\right) \\
& \leq \Phi\left(A\left(\xi_{0}(w), \xi_{1}(w)\right)\right) .
\end{aligned}
$$


(a) If $A\left(\xi_{0}(w), \xi_{1}(w)\right)=0$, then $d\left(F\left(w, \xi_{0}(w)\right), S\left(w, \xi_{0}(w)\right)\right)=0$. By Lemma 1.1, $F\left(w, \xi_{0}(w)\right) \cap S\left(w, \xi_{0}(w)\right) \neq \emptyset$. Taking $s(w) \in F\left(w, \xi_{0}(w)\right) \cap S\left(w, \xi_{0}(w)\right)$, we have

$$
\begin{aligned}
d\left(s(w), T\left(w, \xi_{0}(w)\right)\right) & \leq d\left(s(w), S\left(w, \xi_{0}(w)\right)\right)+H\left(S\left(w, \xi_{0}(w)\right), T\left(w, \xi_{0}(w)\right)\right) \\
& \leq \Phi\left(\operatorname { m a x } \left\{0,0, d\left(F\left(w, \xi_{0}(w)\right), T\left(w, \xi_{0}(w)\right)\right)\right.\right. \\
& \left.\left.\quad d\left(F\left(w, \xi_{0}(w)\right), T\left(w, \xi_{0}(w)\right)\right) / 2\right\}\right) \\
& \leq \Phi\left(d\left(F\left(w, \xi_{0}(w)\right), T\left(w, \xi_{0}(w)\right)\right)\right) \\
& \leq \Phi\left(d\left(s(w), T\left(w, \xi_{0}(w)\right)\right)\right)
\end{aligned}
$$

By Lemma 1.3 (iii) $d\left(s(w), T\left(w, \xi_{0}(w)\right)\right)=0$, since $T\left(w, \xi_{0}(w)\right)$ is closed, $s(w) \in$ $T\left(w, \xi_{0}(w)\right)$. Therefore in this case the conclusion of Theorem 2.2 is proved.

(b) If $A\left(\xi_{0}(w), \xi_{1}(w)\right)>0$, then, by (3) we have

$$
d\left(s_{1}(w), T\left(w, \xi_{1}(w)\right)\right) \leq \Phi\left(A\left(\xi_{0}(w), \xi_{1}(w)\right)\right)<\phi\left(A\left(\xi_{0}(w), \xi_{1}(w)\right)\right)
$$

Consequently, we can find an $s_{2}(w) \in T\left(w, \xi_{1}(w)\right)$ such that

$$
d\left(s_{1}(w), s_{2}(w)\right) \leq \phi\left(A\left(\xi_{0}(w), \xi_{1}(w)\right)\right)
$$

Since $T(w, X) \subset F(w, X)$, for $s_{2}(w) \in T\left(w, \xi_{1}(w)\right) \subset F(w, X)$, there exists a measurable map, say $\xi_{2}: \Omega \rightarrow X$ such that $s_{2}(w) \in F\left(w, \xi_{2}(w)\right)$. This implies that we can find an $s_{2}(w) \in F\left(w, \xi_{2}(w)\right) \cap T\left(w, \xi_{1}(w)\right)$ such that (6) holds.

On the other hand, by the assumption we have

$$
d\left(S\left(w, \xi_{2}(w)\right), s_{2}(w)\right) \leq H\left(S\left(w, \xi_{2}(w)\right), T\left(w, \xi_{1}(w)\right)\right) \leq \Phi\left(A\left(\xi_{2}(w), \xi_{1}(w)\right)\right)
$$

If $A\left(\xi_{2}(w), \xi_{1}(w)\right)=0$, by the same reason as stated in the proof of (a) we can prove that the conclusion of Theorem 2.2 is true.

If $A\left(\xi_{2}(w), \xi_{1}(w)\right)>0$, repeating the reasoning as mentioned above, we can find measurable maps $\xi_{3}, s_{3}: \Omega \rightarrow X$ such that $s_{3}(w) \in F\left(w, \xi_{3}(w)\right) \cap S\left(w, \xi_{2}(w)\right)$ and

$$
d\left(s_{3}(w), s_{2}(w)\right) \leq \phi\left(A\left(\xi_{2}(w), \xi_{1}(w)\right)\right) .
$$

Inductively, we can define two sequence $\left\{\xi_{n}(w)\right\},\left\{s_{n}(w)\right\} \subset X$ such that

$$
\left\{\begin{array}{l}
s_{2 n+1}(w) \in F\left(w, \xi_{2 n+1}(w)\right) \cap S\left(w, \xi_{2 n}(w)\right) \\
s_{2 n+2}(w) \in F\left(w, \xi_{2 n+2}(w)\right) \cap T\left(w, \xi_{2 n+1}(w)\right)
\end{array} \quad n=0,1,2, \ldots\right.
$$

and

$$
\left\{\begin{array}{l}
d\left(s_{2 n+1}(w), s_{2 n+2}(w)\right) \leq \phi\left(A\left(\xi_{2 n}(w), \xi_{2 n+1}(w)\right)\right) \\
d\left(s_{2 n+3}(w), s_{2 n+2}(w)\right) \leq \phi\left(A\left(\xi_{2 n+2}(w), \xi_{2 n+1}(w)\right)\right)
\end{array} \quad n=0,1,2, \ldots\right.
$$


Now, we prove that $\left\{s_{n}(w)\right\}$ is a Cauchy sequence in $X$. In fact, for any positive integer $n$, we have

$$
\begin{aligned}
& A\left(\xi_{2 n}(w), \xi_{2 n+1}(w)\right) \leq \max \{ d\left(s_{2 n}(w), s_{2 n+1}(w)\right), d\left(s_{2 n}(w), s_{2 n+1}(w)\right), \\
& d\left(s_{2 n+1}(w), s_{2 n+2}(w)\right), \\
& {\left.\left[d\left(s_{2 n}(w), s_{2 n+2}(w)\right)+d\left(s_{2 n+1}(w), s_{2 n+1}(w)\right)\right] / 2\right\} } \\
& \leq \max \left\{d\left(s_{2 n}(w), s_{2 n+1}(w)\right), d\left(s_{2 n+1}(w), s_{2 n+2}(w)\right)\right\} .
\end{aligned}
$$

Using the same argument we can prove that

$$
A\left(\xi_{2 n+2}(w), \xi_{2 n+1}(w)\right) \leq \max \left\{d\left(s_{2 n+1}(w), s_{2 n+2}(w)\right), d\left(s_{2 n+2}(w), s_{2 n+3}(w)\right)\right\} .
$$

Consequently, in general, for $n=1,2, \ldots$, we have

$$
\begin{aligned}
d\left(s_{n+1}(w), s_{n+2}(w)\right) & \leq \phi\left(A\left(\xi_{n}(w), \xi_{n+1}(w)\right)\right) \\
& \leq \phi\left(\max \left\{d\left(s_{n}(w), s_{n+1}(w)\right), d\left(s_{n+1}(w), s_{n+2}(w)\right)\right\}\right)
\end{aligned}
$$

If $d\left(s_{n+1}(w), s_{n+2}(w)\right)>d\left(s_{n}(w), s_{n+1}(w)\right) \geq 0$, then, by (9) and Lemma 1.3, we have

$$
d\left(s_{n+1}(w), s_{n+2}(w)\right) \leq \phi\left(d\left(s_{n+1}(w), s_{n+2}(w)\right)\right)<d\left(s_{n+1}(w), s_{n+2}(w)\right)
$$

a contradiction. Therefore, $d\left(s_{n+1}(w), s_{n+2}(w)\right) \leq d\left(s_{n}(w), s_{n+1}(w)\right)$. Hence

$$
d\left(s_{n}(w), s_{n+1}(w)\right) \leq \phi\left(d\left(s_{n-1}(w), s_{n}(w)\right)\right) \leq \cdots \leq \phi^{n-1}\left(d\left(s_{1}(w), s_{2}(w)\right)\right) .
$$

If $d\left(s_{1}(w), s_{2}(w)\right)=0$, that is, $s_{1}(w)=s_{2}(w)$, for all $w \in \Omega$, denoting $s(w)=$ $s_{1}(w)=s_{2}(w)$, then $s(w)=s_{1}(w) \in F\left(w, \xi_{1}(w)\right) \cap S\left(w, \xi_{0}(w)\right), s(w)=s_{2}(w) \in$ $F\left(w, \xi_{2}(w)\right) \cap T\left(w, \xi_{1}(w)\right)$. Hence $s(w) \in F\left(w, \xi_{1}(w)\right) \cap T\left(w, \xi_{1}(w)\right)$. Similarly using the proof in (a) we can prove that $s(w) \in S\left(w, \xi_{1}(w)\right)$. Hence the conclusion of Theorem 2.2 is proved.

If $d\left(s_{1}(w), s_{2}(w)\right)>0$, in view of condition (4), we know that $\sum \phi^{n-1} d\left(s_{1}(w)\right.$, $\left.s_{2}(w)\right)$ is convergent. It follows from (10) that $\sum d\left(s_{n}(w), s_{n+1}(w)\right)$ is convergent too. This implies that $\left\{s_{n}(w)\right\}$ is a Cauchy sequence in $X$. Since $X$ is complete, there exists a measurable map $s^{*}: \Omega \rightarrow X$ such that $s_{n}(w) \rightarrow s^{*}(w)$. Since $s_{n}(w) \epsilon$ $F\left(w, \xi_{n}(w)\right) \subset F(w, X)$ and $F(w, X)$ is closed, this shows that $s^{*}(w) \in F(w, X)$. Hence there exists measurable map $s: \Omega \rightarrow X$ such that $s^{*}(w) \in F(w, s(w))$. By (5) and (7) we have

$$
\begin{aligned}
d\left(s^{*}(w), S(w, s(w))\right) \leq & d\left(s^{*}(w), s_{2 n+2}(w)\right)+d\left(s_{2 n+2}(w), S(w, s(w))\right) \\
\leq & d\left(s^{*}(w), s_{2 n+2}(w)\right)+d\left(s_{2 n+2}(w), T\left(w, \xi_{2 n+1}(w)\right)\right) \\
& +H\left(T\left(w, \xi_{2 n+1}(w)\right), S(w, s(w))\right)
\end{aligned}
$$




$$
\begin{aligned}
d\left(s^{*}(w), S(w, s(w))\right) \leq & d\left(s^{*}(w), s_{2 n+2}(w)\right) \\
+ & \Phi\left(\operatorname { m a x } \left(d\left(s^{*}(w), s_{2 n+1}(w)\right), d\left(s^{*}(w), S(w, s(w))\right),\right.\right. \\
& d\left(s_{2 n+1}(w), s_{2 n+2}(w)\right), \\
& {\left.\left.\left[d\left(s^{*}(w), s_{2 n+2}(w)\right)+d\left(s_{2 n+1}(w), S(w, s(w))\right)\right] / 2\right\}\right) . }
\end{aligned}
$$

Letting $n \rightarrow \infty$, we have $d\left(s^{*}(w), S(w, s(w))\right) \leq \Phi\left(d\left(s^{*}(w), S(w, s(w))\right)\right)$. By Lemma 1.3 (iii) we have $d\left(s^{*}(w), S(w, s(w))\right)=0$. Since $S(w, s(w))$ is closed, we have $s^{*}(w) \in S(w, s(w))$. Similarly, we can prove that $s^{*}(w) \in T(w, s(w))$. Therefore we have $s^{*}(w) \in F(w, s(w)) \cap S(w, s(w)) \cap T(w, s(w))$. This completes the proof.

REMARK. Theorem 2.1 is a special case of Theorem 2.2 with $F$ being a singlevalued mapping and $\Phi(t)=\alpha(w) t$, where $\alpha: \Omega \rightarrow(0,1)$ is a measurable mapping and $t \in \mathbb{R}^{+}$.

COROLlaRY 2.3. Let $T_{i}: \Omega \times X \rightarrow C B(X), i=1,2, \ldots$, be multifunction such that

(i) $T_{i}(w, \cdot), T_{j}(w, \cdot)$ are continuous for all $w \in \Omega, i \neq j$;

(ii) $T_{i}(\cdot, x), T_{j}(\cdot, x)$ are measurable for all $x \in X, i \neq j$;

(iii) For all $i, j, i \neq j$

$$
\begin{gathered}
H\left(T_{i}(w, x), T_{j}(w, y)\right) \leq \Phi\left(\operatorname { m a x } \left\{d(x, y), d\left(x, T_{i}(w, x)\right), d\left(y, T_{j}(w, y)\right),\right.\right. \\
\left.\left.\left[d\left(x, T_{j}(w, y)\right)+d\left(y, T_{i}(w, x)\right)\right] / 2\right\}\right)
\end{gathered}
$$

for all $x, y \in X$ and for all $w \in \Omega$, where $\Phi: \mathbb{R}^{+} \rightarrow \mathbb{R}^{+}$is an increasing function satisfying conditions (1) and (2).

Then the random fixed point sets $\left\{\xi: \Omega \rightarrow X: \xi(w) \in T_{i}(w, \xi(w))\right\}, i=1,2, \ldots$, are nonempty, closed and equal to each other.

PROOF. For the sake of convenience we prove the conclusions of Corollary 2.3 only for the case $i=1$ and $j=2$. By Theorem 2.2, there exists a measurable map $s: \Omega \rightarrow X$ such that $s(w) \in T_{1}(w, s(w)) \cap T_{2}(w, s(w))$. Now we prove that the random fixed point sets of $T_{1}$ and $T_{2}$ are equal to each other. In fact, if measurable map $u: \Omega \rightarrow X$ is a random fixed point of $T_{1}$, that is $u(w) \in T_{1}(w, u(w))$, then we have

$$
\begin{aligned}
d(u(w), & \left.T_{2}(w, u(w))\right) \\
\leq & H\left(T_{1}(w, u(w)), T_{2}(w, u(w))\right) \\
\leq & \Phi\left(\operatorname { m a x } \left\{d(u(w), u(w)), d\left(u(w), T_{1}(w, u(w))\right), d\left(u(w), T_{2}(w, u(w))\right),\right.\right. \\
& {\left.\left.\left[d\left(u(w), T_{2}(w, u(w))\right)+d\left(u(w), T_{1}(w, u(w))\right)\right] / 2\right\}\right) } \\
\leq & \Phi\left(d\left(u(w), T_{2}(w, u(w))\right)\right) .
\end{aligned}
$$


By Lemma 1.3 (iii), we have $d\left(u(w), T_{2}(w, u(w))\right)=0$. Since $T_{2}(w, u(w))$ is closed, $u(w) \in T_{2}(w, u(w))$. Using the same argument we can prove that if a measurable map $v: \Omega \rightarrow X$ is a random fixed point of $T_{2}$ then $v$ is also a random fixed point of $T_{1}$. Hence

$$
\left\{\xi: \Omega \rightarrow X: \xi(w) \in T_{1}(w, \xi(w))\right\}=\left\{\xi: \Omega \rightarrow X: \xi(w) \in T_{2}(w, \xi(w))\right\} .
$$

Let $\left\{\xi_{n}(w)\right\} \subset\left\{\xi: \Omega \rightarrow X: \xi(w) \in T_{1}(w, \xi(w))\right\}$ and $\xi_{n}(w) \rightarrow \xi(w)$ as $n \rightarrow \infty$. Since $\xi_{n}(w) \in T_{1}\left(w, \xi_{n}(w)\right)$ and $T_{1}\left(w, \xi_{n}(w)\right) \rightarrow T_{1}(w, \xi(w))$ as $n \rightarrow \infty$. We have

$$
\begin{aligned}
d\left(\xi(w), T_{1}(w, \xi(w))\right) & \leq d\left(\xi(w), \xi_{n}(w)\right)+d\left(\xi_{n}(w), T_{1}(w, \xi(w))\right) \\
& \leq d\left(\xi(w), \xi_{n}(w)\right)+H\left(T_{1}\left(w, \xi_{n}(w)\right), T_{1}\left(w, \xi_{1}(w)\right)\right) \stackrel{n \rightarrow \infty}{\longrightarrow} 0
\end{aligned}
$$

that is, $\xi(w) \in T_{1}(w, \xi(w))$. Therefore, $\left\{\xi: \Omega \rightarrow X: \xi(w) \in T_{1}(w, \xi(w))\right\}$ is closed. This completes the proof.

Let $S, T: \Omega \times X \rightarrow C B(X)$ and $F: \Omega \times X \rightarrow C C(X)$ be multifunctions such that

$$
\begin{aligned}
& H(S(w, x), T(w, y)) \leq \Psi(d(F(w, x), F(w, y)), d(F(w, x), S(w, x)), \\
& d(F(w, y), T(w, y)), d(F(w, x), T(w, y)), \\
& d(F(w, y), S(w, x)))
\end{aligned}
$$

for all $x, y \in X$ and for all $w \in \Omega$, where $\Psi\left(t_{1}, t_{2}, t_{3}, t_{4}, t_{5}\right): \mathbb{R}^{+5} \rightarrow \mathbb{R}^{+}$satisfies condition $(\Psi)$.

THEOREM 2.4. Let $S, T: \Omega \times X \rightarrow C B(X)$ and $F: \Omega \times X \rightarrow C C(X)$ be multifunctions such that

(i) $S(w, \cdot), T(w, \cdot)$ are both continuous for all $w \in \Omega$;

(ii) $S(\cdot, x), T(\cdot, x)$ are both measurable for all $x \in X$;

(iii) $S(w, X) \cup T(w, X) \subset F(w, X), F(w, X)$ is closed;

(iv) $S, T$ and $F$ satisfy (12) for all $w \in \Omega$ and all $x, y \in X$.

Then there exists a measurable map $s: \Omega \rightarrow X$ such that

$$
F(w, s(w)) \cap S(w, s(w)) \cap T(w, s(w)) \neq \emptyset .
$$

ProOF. Let

$$
\begin{gathered}
t^{*}=\max \{d(F(w, x), F(w, y)), d(F(w, x), S(w, x)), d(F(w, y), T(w, y)), \\
[d(F(w, x), T(w, y))+d(F(w, y), S(w, x))] / 2\}
\end{gathered}
$$


Without loss of generality we can assume that

$$
d(F(w, x), T(w, y)) \geq d(F(w, y), S(w, x)) .
$$

Then

$$
\begin{aligned}
t^{*} & \geq \max \{d(F(w, x), F(w, y)), d(F(w, x), S(w, x)), d(F(w, y), T(w, y))\}, \\
t^{*} & \geq[d(F(w, x), T(w, y))+d(F(w, y), S(w, x))] / 2 \geq d(F(w, y), S(w, x)), \\
2 t^{*} & \geq d(F(w, x), T(w, y))+d(F(w, y), S(w, x)) \geq d(F(w, x), T(w, y)) .
\end{aligned}
$$

Using condition $(\Psi)$ and (12) we have

$$
\begin{aligned}
H(S(w, x), T(w, y)) \leq & \Psi\left(t^{*}, t^{*}, t^{*}, 2 t^{*}, t^{*}\right) \leq \Phi\left(t^{*}\right) \\
= & \Phi(\max \{d(F(w, x), F(w, y)), d(F(w, x), S(w, x)), \\
& d(F(w, y), T(w, y)), \\
& {[d(F(w, x), T(w, y))+d(F(w, y), S(w, x))] / 2\}) . }
\end{aligned}
$$

Therefore, $F, S$ and $T$ satisfy all conditions of Theorem 2.2. The conclusion of Theorem 2.4 follows from Theorem 2.2 immediately.

From Theorem 2.4 we can obtain the following

COROllaRY 2.5. Let $T_{i}: \Omega \times X \rightarrow C B(X), i=1,2, \ldots$, be multifunctions such that

(i) $T_{i}(w, \cdot), T_{j}(w, \cdot)$ are continuous for all $w \in \Omega, i \neq j$;

(ii) $T_{i}(\cdot, x), T_{j}(\cdot, x)$ are measurable for all $x \in X, i \neq j$;

(iii) For all $i, j, i \neq j$

$$
\begin{gathered}
H\left(T_{i}(w, x), T_{j}(w, y)\right) \leq \Psi\left(d(x, y), d\left(x, T_{i}(w, x)\right), d\left(y, T_{j}(w, y)\right),\right. \\
\left.d\left(x, T_{j}(w, y)\right), d\left(y, T_{i}(w, x)\right)\right),
\end{gathered}
$$

for all $w \in \Omega$ and all $x, y \in X$, where $\Psi\left(t_{1}, t_{2}, t_{3}, t_{4}, t_{5}\right): \mathbb{R}^{+5} \rightarrow \mathbb{R}^{+}$satisfies condition $(\Psi)$.

Then the random fixed point sets $\left\{\xi: \Omega \rightarrow X: \xi(w) \in T_{i}(w, \xi(w))\right\}, i=1,2, \ldots$, are nonempty, closed and equal to each other.

REMARK. Our results are stochastic versions of the corresponding results of Chang [2].

\section{Acknowledgements}

The author would like to express his sincere thanks to the referee for his valuable suggestions leading to the improved presentation of this paper. The author would 
also like to express his gratitude to professor Chen Falai for financial support and to Professor Jin Liang for his helpful suggestions.

This work is supported by the Outstanding Youth Grant of NSF of China (No. 60225002), NKBRSF on Mathematical Mechanics (No. G1998030600), the TRAPOYT in Higher Education Institute of MOE of China and the Doctoral Program of MOE of China (No. 20010358003).

\section{References}

[1] C. Adrian, 'A random fixed point theorem for multifunctions', Stochastic Anal. Appl. (1) 12 (1994), 65-73.

[2] S. S. Chang and Y. C. Peng, 'Coincidence point theorems for multivalued mappings', Internat. J. Math. Math. Sci. (1) 16 (1993), 87-94.

[3] C. J. Himmelberg, 'Measurable relations', Fund. Math. 87 (1975), 53-72.

[4] H. Kaneko and S. Sessa, 'Fixed point theorems for compactible multivalued and single valued mappings', Internat. J. Math. Math. Sci. (2) 12 (1989), 257-262.

[5] T. Kubiak, 'Fixed point theorems for contrctive type multivalued mappings', Math. Japonica 30 (1985), 89-101.

[6] K. Kuratowski and C. Rull Nardzewski, 'A general theorem on selectors', Bull. Acad. Polon. Sci. Ser. Sci. Math. Astronom. Phys. 13 (1965), 397-403.

[7] G. Mustafa, 'Random coincidence point theorems for Kannan type random multi-valued operators on polish spaces', J. Pure Appl. Sci. (2) 19 (2000), 135-140.

[8] — , 'Some random coincidence point theorems', J. Math. Research Exposition 23 (2003), $413-421$.

[9] G. Mustafa and H. Nawab, 'Random coincidence points of random multivalued operators on polish spaces', J. Pure Appl. Sci. (2) 17 (1998), 58-61.

[10] - 'Random coincidence point theorems for generalized contractive type random multivalued operators on polish spaces', J. Pure Appl. Sci. (1) 19 (2000), 17-23.

[11] N. S. Papageorgiou, 'Random fixed point theorems for multifunctions', Math. Japonica 29 (1984), 93-106.

[12] R. T. Rockafellar, 'Measurable dependence of convex sets and functions in parameters', J. Math. Anal. Appl. 28 (1969), 4-25.

[13] K. P. R. Sastry, S. V. R. Naidu and J. R. Prasad, 'Common fixed points for multimaps in a metric space', Nonlinear Anal. 13 (1989), 221-229.

\section{Department of Mathematics}

University of Science and Technology of China

Hefei Anhui 230026 P.R. China

and Islamia University Bahawal pur Pakistan

e-mail: mustafa_rakib@yahoo.com 